\title{
Acceleration and Pickup Ring of Energetic Electrons Observed in Relativistic Magnetic Reconnection Simulations
}

\author{
Y. L. Ping ${ }^{1,2}$, J. Y. Zhong ${ }^{2,3}$, X. G. Wang ${ }^{4}$, Z. M. Sheng ${ }^{3,5,6}$ (i) , and G. Zhao ${ }^{1}$ \\ ${ }^{1}$ Key Laboratory of Optical Astronomy, National Astronomical Observatories, Chinese Academy of Sciences, Beijing 100012, China; ylping@bao.ac.cn, \\ gzhao@bao.ac.cn \\ ${ }^{2}$ Department of Astronomy, Beijing Normal University, Beijing 100875, China; jyzhong@ @nu.edu.cn \\ ${ }^{3}$ MOE Key Laboratory for Laser Plasmas and Collaborative Innovation Center of IFSA, Shanghai Jiao Tong University, Shanghai 200240, China \\ ${ }^{4}$ Department of Physics, Harbin Institute of Technology, Harbin 150001, China \\ ${ }^{5}$ School of Physics and Astronomy and Tsung-Dao Lee Institute, Shanghai Jiao Tong University, Shanghai 200240, China \\ ${ }^{6}$ Department of Physics, SUPA, University of Strathclyde, Glasgow G4 0NG, UK \\ Received 2017 January 4; revised 2017 September 16; accepted 2017 September 21; published 2017 November 8
}

\begin{abstract}
Pickup ring of energetic electrons found in relativistic magnetic reconnection (MR) driven by two relativistic intense femtosecond laser pulses is investigated by particle simulation in 3D geometry. Magnetic reconnection processes and configurations are characterized by plasma current density distributions at different axial positions. Two helical structures associated with the circular polarization of laser pulses break down in the reconnection processes to form a current sheet between them, where energetic electrons are found to pile up and the outflow relativistic electron jets are observed. In the field line diffusion region, electrons are accelerated to multi-MeV with a flatter power-law spectrum due to MR. The development of the pickup ring of energetic electrons is strongly dependent upon laser peak intensities.
\end{abstract}

Key words: acceleration of particles - magnetic reconnection - methods: numerical - plasmas relativistic processes

\section{Introduction}

The origins of the nonthermal radiation of gamma-ray bursts and jets of active galactic nuclei (AGNs) are outstanding issues of astrophysics (Waxman 2006; Nakar 2007). It has been proposed that such nonthermal radiation could be produced from ultrarelativistic jet outflows picking up ambient interstellar protons and electrons (Schlickeiser \& Dermer 2000; Schlickeiser et al. 2002, 2003; Gerbig \& Schlickeiser 2007).

Magnetic reconnection (MR; Biskamp 2000; Yamada et al. 2010) is known as a fundamental mechanism for fast energy conversion from magnetic fields into particle energies. It is supposed to be responsible for energetic electron generation and high-frequency radiations in processes such as solar flares (Parker 1957; Sweet 1958), coronal mass ejections, interaction of the solar wind and magnetosphere (Brady et al. 2009), as well as relativistic objects (Matteo 1998; Lyubarsky \& Kirk 2001; McKinney \& Uzdensky 2012) and fusion plasma instabilities (Taylor 1986). Particularly, particle acceleration to the relativistic regime in such energy conversion processes is essentially important for impulsive and/or fierce space, laboratory, and astrophysical plasma phenomena (Lin et al. 2003; Zhang \& Mészáros 2004; Drake et al. 2005, 2006). Recently, due to the development of high power laser technology, there is increasing interest in strongly driven fast reconnection processes in laboratory with laser pulses, which can simulate impulsive astrophysical events (Nilson et al. 2006; Li et al. 2007; Zhong et al. 2010).

With relativistic intense lasers, quasi-static magnetic fields of hundreds of Mega-Gausses may be generated, as shown numerically (Pukhov \& Meyer-ter-Vehn 1996; Fuchs et al. 1998; Tanimoto et al. 2003) and theoretically (Sheng \& Meyer-ter-Vehn 1996; Qiao et al. 2005) in plasmas at a moderate density. Even higher magnetic fields near giga-Gauss have also been measured in intense laser interaction with solid targets, where the region of strong magnetic fields is located both at the surface and inside the target (Wagner et al. 2004). Magnetic reconnection has been foreseen in such laserproduced plasmas (Askar'yan et al. 1997). In the ultra-intense laser and near-critical plasma conditions, many novel phenomena have been extensively studied, such as relativistic magnetic self-channeling of light (Pukhov \& Meyer-ter-Vehn 1996), magnetic-dipole vortex generation (Nakamura \& Mima 2008), and so on. Recently, it has been found that the self-generated quasi-static axial magnetic field by a circularly polarized laser can be strong enough to pinch and trap the relativistic thermal electrons in the center of a plasma channel produced by the laser (Liu et al. 2013). Also an MR process in self-generated magnetic fields produced by two parallel incident laser beams shooting into a near-critical plasma layer was recently investigated numerically in three-dimensional (3D) geometry (Ping et al. 2014). It is found that, instead of the electromagnetic turbulence effect, the electrostatic turbulence contribution to the reconnecting electric field plays an essential role in the process.

In this paper, we report a pickup ring of energetic electrons observed in numerical simulation of relativistic MR driven by two intense femtosecond laser pulses in a dense plasma. The spatio-temporal evolution of distributions of relativistic electrons due to reconnection and resulting energy spectra of these electrons are also revealed.

\section{Simulation Setup and Reconnection}

In the simulation, a fully relativistic particle-in-cell (PIC) code (KLAP; Chen et al. 2008; Yan et al. 2008) is applied. The simulation box has a size of $L_{x 0}=L_{y 0}=24 \mu \mathrm{m}$ and $L_{z 0}=50 \mu \mathrm{m}$, with $480 \times 480 \times 1000$ cells and eight particles per cell on average. The region of $5 \mu \mathrm{m}<$ $z<10 \mu \mathrm{m}$ is full of a dense electron-proton plasma with its 

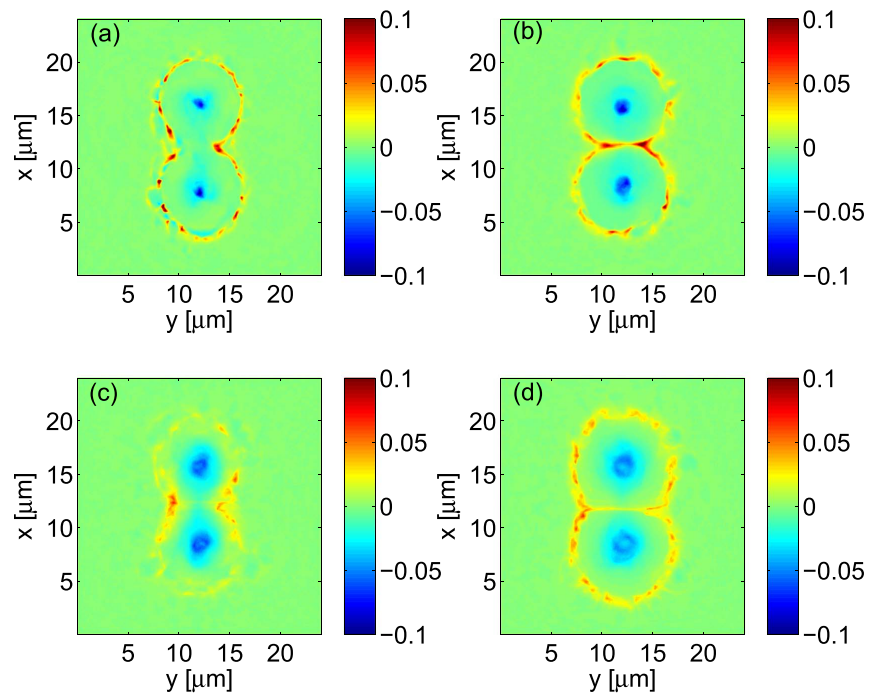

Figure 1. Distributions of the axial current density $j_{z}$ at different positions of $z=15 \mu \mathrm{m}$ (a), $20 \mu \mathrm{m}$ (b), $24 \mu \mathrm{m}$ (c), and $27.5 \mu \mathrm{m}$ (d), corresponding to the locations marked by vertical lines in Figure 2(d), at $t=50 T_{0}$.

density linearly increasing to the critical value of $n_{c}=$ $m_{e} \omega_{0}^{2} / 4 \pi e^{2}=1.15 \times 10^{21} \mathrm{~cm}^{-3}$ at $10 \mu \mathrm{m}$, where $m_{e}$ is the electron rest mass, $\omega_{0}$ is the incident laser angular frequency, and $e$ is the element charge. Then the plasma density remains to be at critical density in $10 \mu \mathrm{m}<z<15 \mu \mathrm{m}$. In the region of $15 \mu \mathrm{m}<z<35 \mu \mathrm{m}$, the plasma density linearly decreases as $n(z)=\left(1-\left(z-z_{0}\right) / L_{0}\right) n_{c}$, where $L_{0}=20 \mu \mathrm{m}$ and $z_{0}=$ $15 \mu \mathrm{m}$. The initial electrons and ion temperatures are 10 and $0.01 \mathrm{keV}$, respectively. Two identical circularly polarized laser beams shoot at the target and then propagate in parallel (along the $z$-direction) in the plasma, with a centroids separation of $8 \mu \mathrm{m}$. The lasers have a peak intensity of $5 \times 10^{20} \mathrm{~W} \mathrm{~cm}^{-2}$, corresponding to a normalized laser vector potential of $a_{0}=13.5$. It has a spot diameter of $3 \mu \mathrm{m}$, and a wavelength of $\lambda_{0}=1 \mu \mathrm{m}$, corresponding to a period of $T_{0}=\lambda_{0} / c=3.33 f s$. The electron skin depth is then $d_{e}=c / \omega_{p e} \approx 0.71 \mu \mathrm{m} \sim \lambda_{0}$, where the electron plasma frequency is $\omega_{p e}=\left(4 \pi e^{2} n_{0} / \gamma_{0} m_{e}\right)^{-1 / 2}$ with the relativistic factor $\gamma_{0} \approx\left(1+a_{0}^{2}\right)^{1 / 2}$. Furthermore, the electron Debye length is $\lambda_{D}=\sqrt{k T_{e} / 4 \pi n_{e} e^{2}} \approx 0.235 \mu \mathrm{m} \approx 4.7 l$, where $l=0.05 \mu \mathrm{m}$ as the size of a cell in the simulation box. The incident laser pulse has a rising front of two laser cycles, followed by a flat top in the $z$-direction and is in a Gaussian form in $x, y$-directions.

The $x-y$ plane cross-section configurations of MR are presented by the axial plasma current density $j_{z}$ distributions at four different axial positions of $z=15 \mu \mathrm{m}, 20 \mu \mathrm{m}$, $24 \mu \mathrm{m}$, and $27.5 \mu \mathrm{m}$, respectively, as shown in Figure 1, corresponding to the vertical black lines in Figure 2(d), at $t=50 T_{0}$. It is found that the circle-like current distribution contours generated by each single laser pulse are attached to each other, broken, and reconnected, with a current sheet being created in the reconnection region. Particularly at $z=15 \mu \mathrm{m}$ (Figure 1(a)) and $24 \mu \mathrm{m}$ (Figure 1(c)), the current sheet clearly has a Sweet-Parker-like Y-type geometry, indicating the presence of a strongly driven MR process.

To further explore the three-dimensionality of MR, the 3D isosurface distributions for the electron energy density of
$200 n_{c} m_{e} c^{2}$ and the cross section of $j_{z}$ over the plane of $y=12 \mu \mathrm{m}$ are calculated and shown on the right of Figure 2 (also at $t=50 T_{0}$ ), with the left for the single laser case as a comparison. The isosurface for the single laser case plotted in Figure 2(a) shows a helical structure similar to that found in resonance acceleration of electrons (Liu et al. 2013). In the case with two lasers shown in Figure 2(b), however, the helical structure of the isosurface is destroyed due to MR driven by the two laser pulses. Also, significant relativistic electron population (Figure 2(b)) and current sheet formation (Figure 2(d)), particularly at the vertical line positions of $1(\mathrm{~b}, \mathrm{~d})$ (corresponding to Figures $1(\mathrm{~b}),(\mathrm{d})$ ), are found in the region enclosing the reconnection $X$-point (or $X$-line in 3D) at $x=y=12 \mu \mathrm{m}$.

\section{Pickup Ring of Energetic Electrons by Magnetic Reconnection}

According to the above analysis, we concentrate on electrons located in the regions of $11 \mu \mathrm{m}<x<13 \mu \mathrm{m}$, $7 \mu \mathrm{m}<y<17 \mu \mathrm{m}$, and $10 \mu \mathrm{m}<z<25 \mu \mathrm{m}$, enfolding the reconnection current sheet. The phase space distributions of electrons of $\left(p_{z}, p_{y}\right)$ in the reconnection region at more time steps are shown in Figure $3 \mathrm{~B}$ for $I_{20}=5 \times 10^{20} \mathrm{~W} \mathrm{~cm}^{-2}$. The acceleration along the negative $z$-direction is clearly due to the reconnection electric field $E_{z}$ (in $\left(p_{z}, p_{y}\right)$ ). A novel structure in the phase space of $\left(p_{z}, p_{y}\right)$ is shown in Figure $3 \mathrm{~B}(\mathrm{c})$, corresponding to two peaks of $p_{y}$ in Figure 4(f). This structure is very similar to the pickup ring (though distorted by relativistic effects) of solar wind protons ( $\mathrm{Wu} \&$ Davidson 1972). It indicates that a large portion of low energy electrons are "picked up" by a frame moving in the negative $z$-direction with a velocity of $v_{z} \approx-0.9992 c\left(p_{z} \approx-25\right)$, corresponding to the Alfvén velocity of the electron, $V_{A e} \sim c / \sqrt{\epsilon}$, where $\epsilon=1+2\left(\omega_{p e 0} / \Omega_{c}\right)^{2}$ is the relative permittivity with the electron cyclotron frequency $\Omega_{c}=e B / m_{e} c$ and $\omega_{p e 0}=\left(4 \pi e^{2} n_{0} / m_{e}\right)^{-1 / 2}$. All these features appearing in the MR process become weaker at a later time of $t=60 T_{0}$ (Figure 3B(e)) when the most intensive MR process is over. Particularly, the "pickup ring" (an ellipse in the relativistic regime) is diffused in both momentum and coordinate spaces, as seen in Figures 3B(c), (d), (e). It suggests a quasilinear diffusion mechanism of wave particle resonance.

Then, similar simulations are performed with different laser peak intensities. Figure 3A presents the phase space distributions of electrons of $\left(p_{z}, p_{y}\right)$ and $\left(p_{z}, p_{x}\right)$ for the lasers with a peak intensity $I_{0}=3 \times 10^{20} \mathrm{~W} \mathrm{~cm}^{-2}$. The "pickup ring" is again observed in Figures $3 \mathrm{~A}(\mathrm{c}),(\mathrm{d}),(\mathrm{e})$. Comparing with Figure 3B, where the "pickup ring" has already occurred for the lasers with the stronger $I_{20}=5 \times 10^{20} \mathrm{~W} \mathrm{~cm}^{-2}$, but it does not occur for the lasers with the weaker $I_{10}=3 \times 10^{20} \mathrm{~W} \mathrm{~cm}^{-2}$, until $t=50 T_{0}$.

Figure $3 \mathrm{C}$ presents the phase space distributions of electrons of $\left(p_{z}, p_{y}\right)$ and $\left(p_{z}, p_{x}\right)$ for the lasers with $I_{30}=$ $7 \times 10^{20} \mathrm{~W} \mathrm{~cm}^{-2}$. The "pickup ring" occurs at $t=45 T_{0}$ in Figure $3 \mathrm{C}(\mathrm{b})$. To compare with Figure $3 \mathrm{~A}$, the "pickup ring" is larger at the same time, indicating that the electrons are accelerated to higher energy. The evolution of the pickup ring of energetic electrons shown at different laser peak intensities suggests that it is produced during the MR process rather than due to some instability. 
(a)
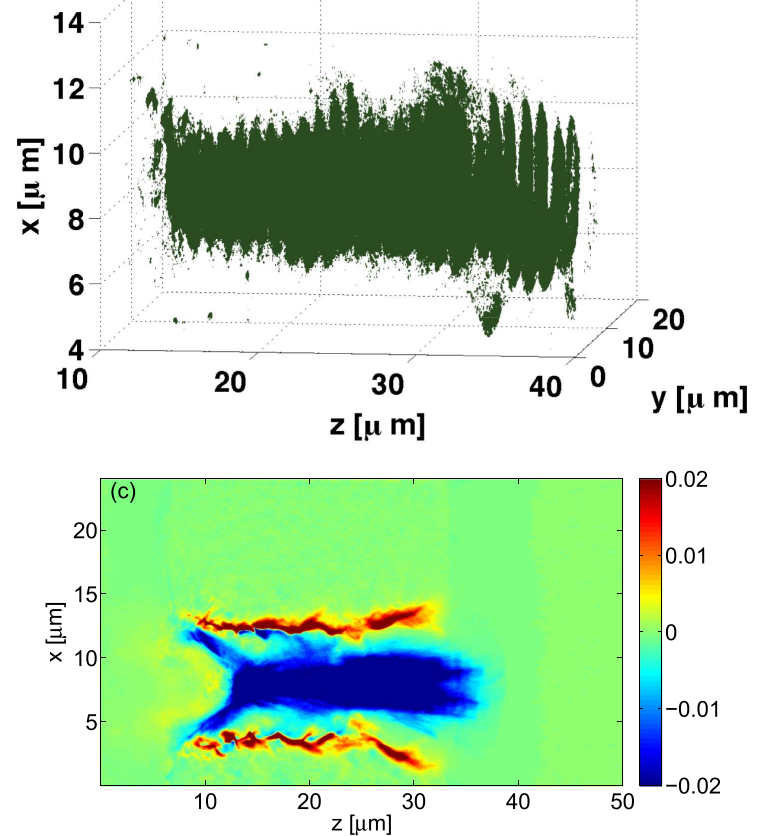

(b)
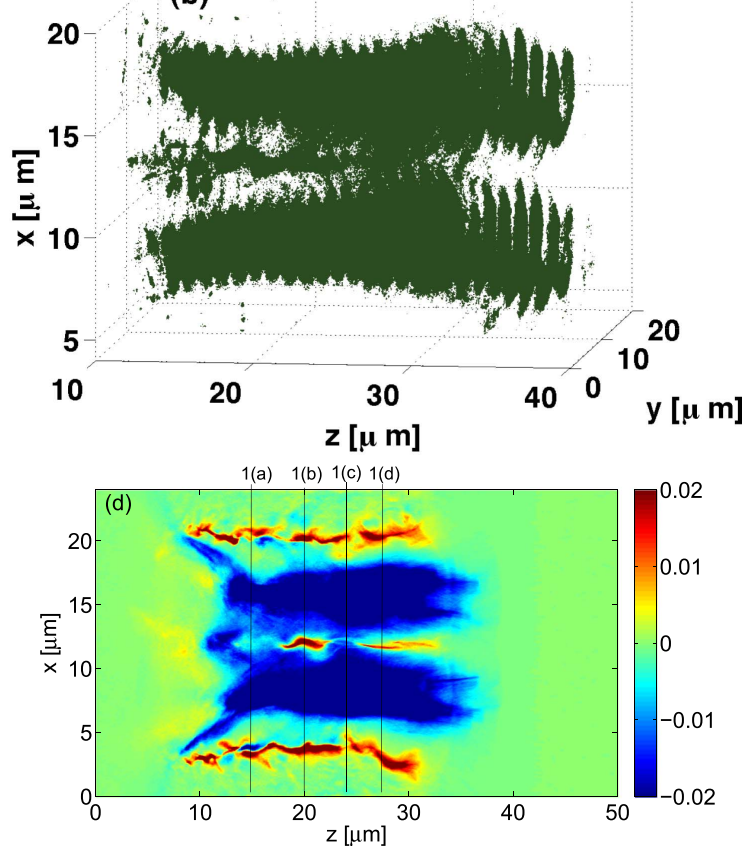

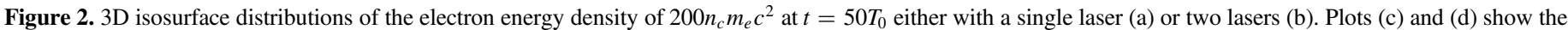

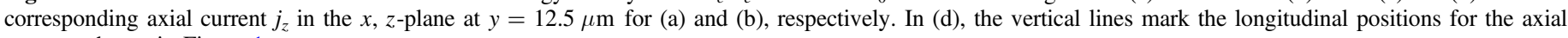
currents shown in Figure 1.
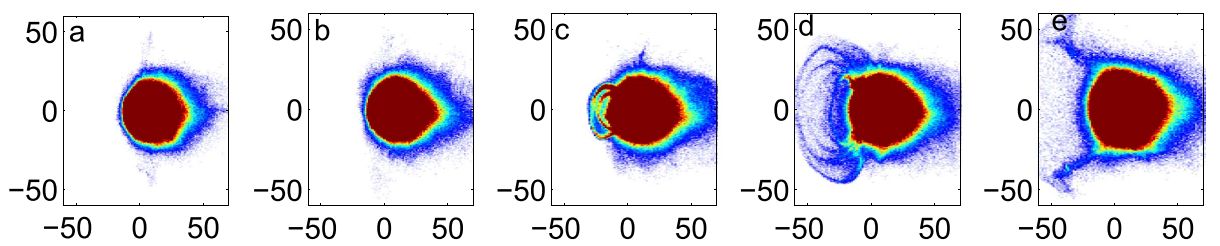

A
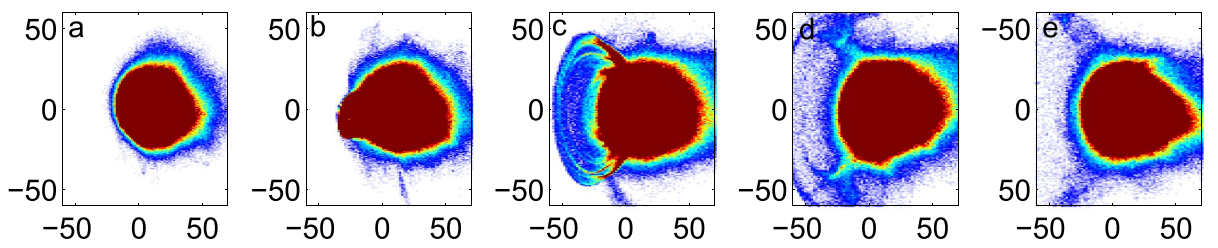

B
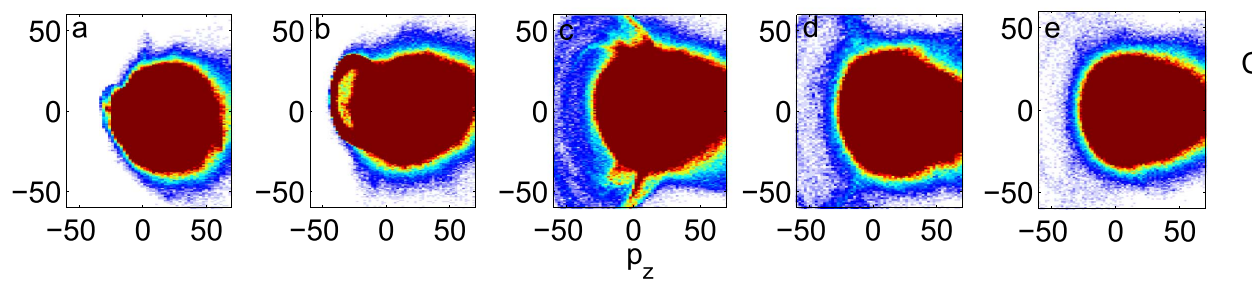

Figure 3. Electron distributions in the phase space of $\left(p_{z}, p_{y}\right)$. From left to right, the columns correspond to the times $t=40 T_{0}, 45 T_{0}, 50 T_{0}, 55 T_{0}$, and $60 T_{0}$, respectively. Row A is for $I_{20}=3 \times 10^{20} \mathrm{~W} \mathrm{~cm}^{-2}$, row B is for $I_{20}=5 \times 10^{20} \mathrm{~W} \mathrm{~cm}^{-2}$, and row $\mathrm{C}$ is for $I_{20}=7 \times 10^{20} \mathrm{~W} \mathrm{~cm}^{-2}$.

\section{Particle Acceleration by Magnetic Reconnection}

In this section, we focus on the electron acceleration in this MR process for $I_{20}=5 \times 10^{20} \mathrm{~W} \mathrm{~cm}^{-2}$. The phase space distributions of electrons in this region at different moments are plotted in Figure 4, where Figures 4(b), (e) are for $t=50 T_{0}$ and Figures 4(c), (f) are for $t=60 T_{0}$, with Figures 4(a), (d) for the one laser case at $t=50 T_{0}$ as a comparison. Comparing Figures 4(b), (e) and 4(a), (d) at $t=50 T_{0}$, the phase space $\left(p_{y}, p_{x}\right)$ indicates that the electron acceleration happens not only in the outflow $y$-direction, but also significantly in the $x$-direction. This is probably due to the outflow along the separatrix at the $Y$-points; moreover, the phase space $\left(y, p_{y}\right)$ shows that the acceleration is asymmetric with a negative $p_{y}$ peak at $\sim y=10 \mu \mathrm{m}$ and a positive $p_{y}$ peak at $y=14 \mu \mathrm{m}$ on two sides of the $X$-line, similar to that in previous PIC simulations with a guide field (Fu et al. 2006; Le et al. 2013). Above features show clearly that the acceleration is essentially along the field lines and caused by the reconnection electric field.

In order to further analyze particle acceleration in the process, the particle energy density distributions averaged over 

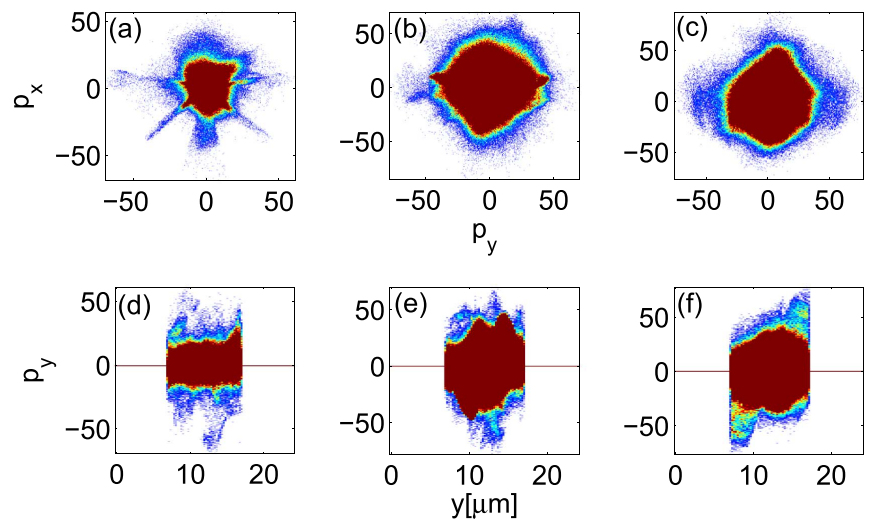

Figure 4. Electron distributions in the phase space of $\left(p_{y}, p_{x}\right)(\mathrm{a}, \mathrm{b}, \mathrm{c})$, and $\left(y, p_{y}\right)(\mathrm{d}, \mathrm{e}, \mathrm{f})$. The $(\mathrm{a}, \mathrm{d})$ are for the one laser case at $t=50 T_{0},(\mathrm{~b}, \mathrm{c}, \mathrm{e}, \mathrm{f})$ are for the two-laser case at $t=50 T_{0}$ and $60 T_{0}$, respectively, $I_{20}=5 \times 10^{20} \mathrm{~W} \mathrm{~cm}^{-2}$, and $p_{i}=\gamma v_{i} / c(i=x, y, z)$.
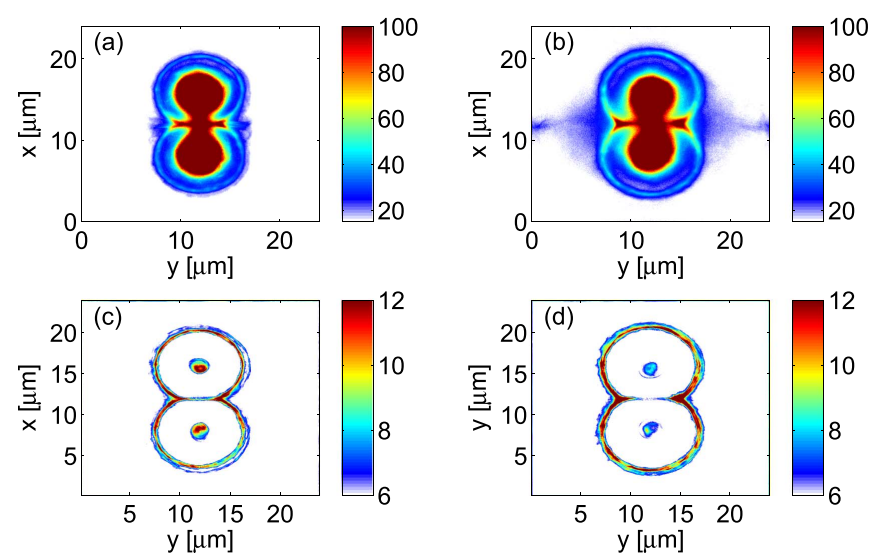

Figure 5. Energy density distributions for the two-laser case with $I_{20}=5 \times 10^{20} \mathrm{~W} \mathrm{~cm}^{-2}$. (a, b) for electrons (scaled by $n_{c} m_{e} c^{2}$ ) and (c, d) for ions (scaled by $n_{c} m_{i} c^{2}$ ). The left and right columns correspond to the time $t=50 T_{0}$ and $60 T_{0}$, respectively.
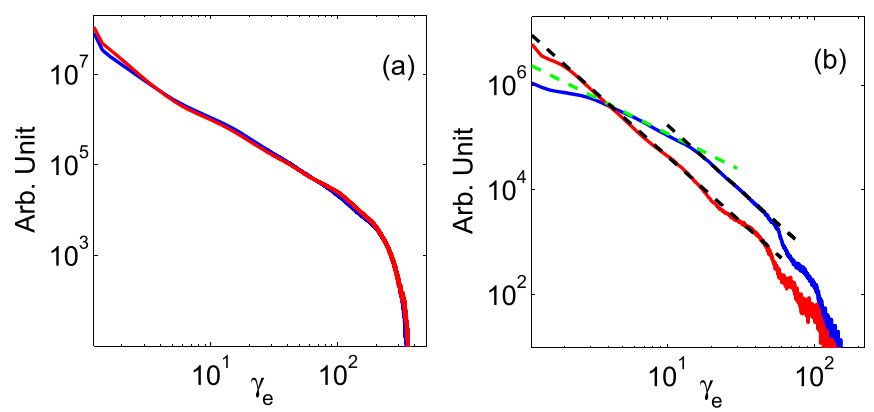

Figure 6. Energy spectra for the electrons in the entire simulation box (a) and in the reconnection region only (b) for $I_{20}=5 \times 10^{20} \mathrm{~W} \mathrm{~cm}^{-2}$. The solid blue curves are for the two-laser case and the red for the one laser case. In (a), the red line has been multiplied by a factor of 2 to compare with the blue line with two lasers. In (b), the dashed lines indicate the power law of the spectrum, $\gamma_{e}^{-p}$, with the black line for $p=2.5$ and the green line for $p=1.4$.

the plasma region along the $z$-direction are given in Figures 5(a), (b) for electrons, and Figures 5(c), (d) for ions in the two-laser case. The electron outflow of reconnection can be clearly seen in Figure 5(a), (b). Comparing Figure 5(b) with Figure 5(a), one can clearly find that electrons are accelerated along newly formed magnetic field lines in the MR process at the later stage. Ions are also accelerated toward the two outflow

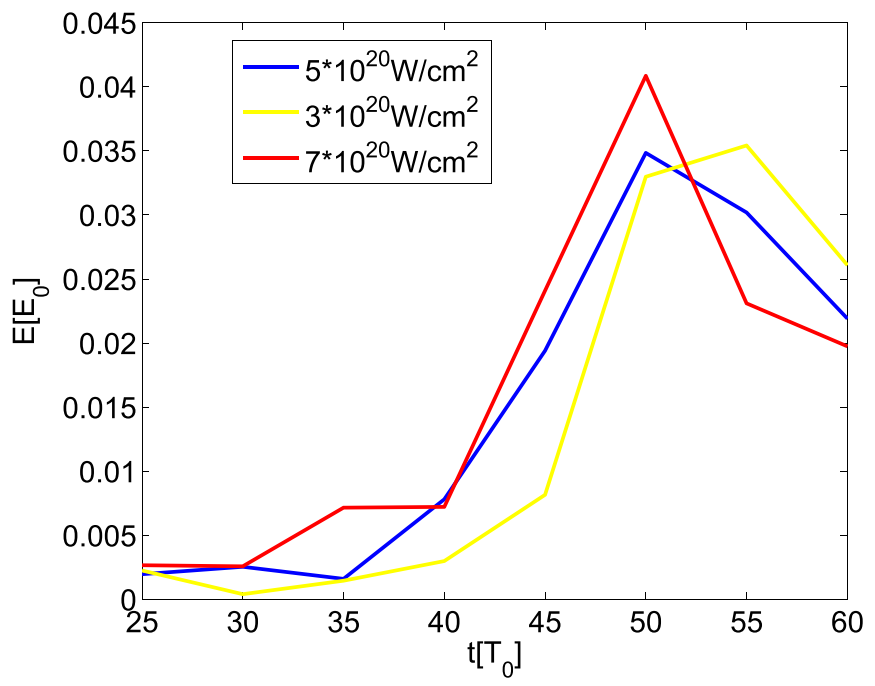

Figure 7. Electric fields are for the laser peak intensity $I_{30}=7 \times$ $10^{20} \mathrm{~W} \mathrm{~cm}^{-2}$ (red line), $I_{20}=5 \times 10^{20} \mathrm{~W} \mathrm{~cm}^{-2}$ (blue line), and $I_{10}=3 \times$ $10^{20} \mathrm{~W} \mathrm{~cm}^{-2}$ (yellow line), respectively. Here, electric fields are normalized by the initial laser field $E_{0}=c B_{0}=4.34 \times 10^{13} \mathrm{~V} / \mathrm{m}$.

directions as shown in Figures 5(c), (d), though not as significantly as electrons due to their larger mass.

The electron energy spectra are presented in Figure 6 for electrons in the entire simulation box and for those in the reconnection region of $11 \mu \mathrm{m}<x<13 \mu \mathrm{m}, 7 \mu \mathrm{m}<$ $y<17 \mu \mathrm{m}$, and $10 \mu \mathrm{m}<z<25 \mu \mathrm{m}$. For the entire electron population, the energy spectra of the two cases, shown in Figure 6(a) are very similar to each other, indicating that the number of electrons accelerated by MR is only a small portion compared with that by direct laser acceleration. Nevertheless, the spectra in the reconnection region, plotted in Figure 6(b), demonstrate substantial acceleration of electrons by MR in the relativistic regime $\left(\gamma_{e}>4\right)$ with an energy gain of $\delta \gamma_{e} \sim 10-100$. Moreover, the spectra for the two cases show different power-law profiles $\gamma_{e}^{p}$. For the one laser case, the power is approximately $p=2.5$ with a knee in the highly relativistic regime $\left(\gamma_{e}>45\right)$. On the other hand, for the case with MR, the spectrum is changed to a power of $p=1.4$ in the $\mathrm{MeV}$ relativistic regime of $4<\gamma_{e}<\gamma_{\text {pon }}$, where $\gamma_{\text {pon }} \sim a_{0}$ is the peak ponderomotive potential of the laser pulse. It recovers the power law for the single laser case with $p=2.5$ in the intermediate relativistic regime of $\gamma_{\text {pon }}<\gamma<50$, nevertheless with an energy gain of $\delta \gamma_{e} \sim 20-40$. In the higher energy regime of the knee region, the energy gain is up to $\delta \gamma_{e} \sim 100$. Thus, the spectra shown in Figure 5(b) demonstrate clearly the electron acceleration by MR in the relativistic regime. Note that the power law of $p=1.4$ below $\gamma_{e}=\gamma_{\text {pon }}$, is similar to the observed gamma-ray burst spectra, which have a flatter slope than that produced by the internal shocks mechanism (Ghisellini et al. 2000; Asano \& Terasawa 2009), though the particle energy in our simulation is still much below the $\mathrm{GeV}$ level of the gamma-ray bursts.

\section{Discussions and Conclusions}

It is worth pointing out that certain crucial parameters in this simulation are in the same regime of AGNs. For instance, The initial electron temperature is $10 \mathrm{keV}$ in the simulation, on the same order of the temperature inferred for AGN jets (Homan et al. 2006). The plasma beta is the ratio of the plasma pressure 

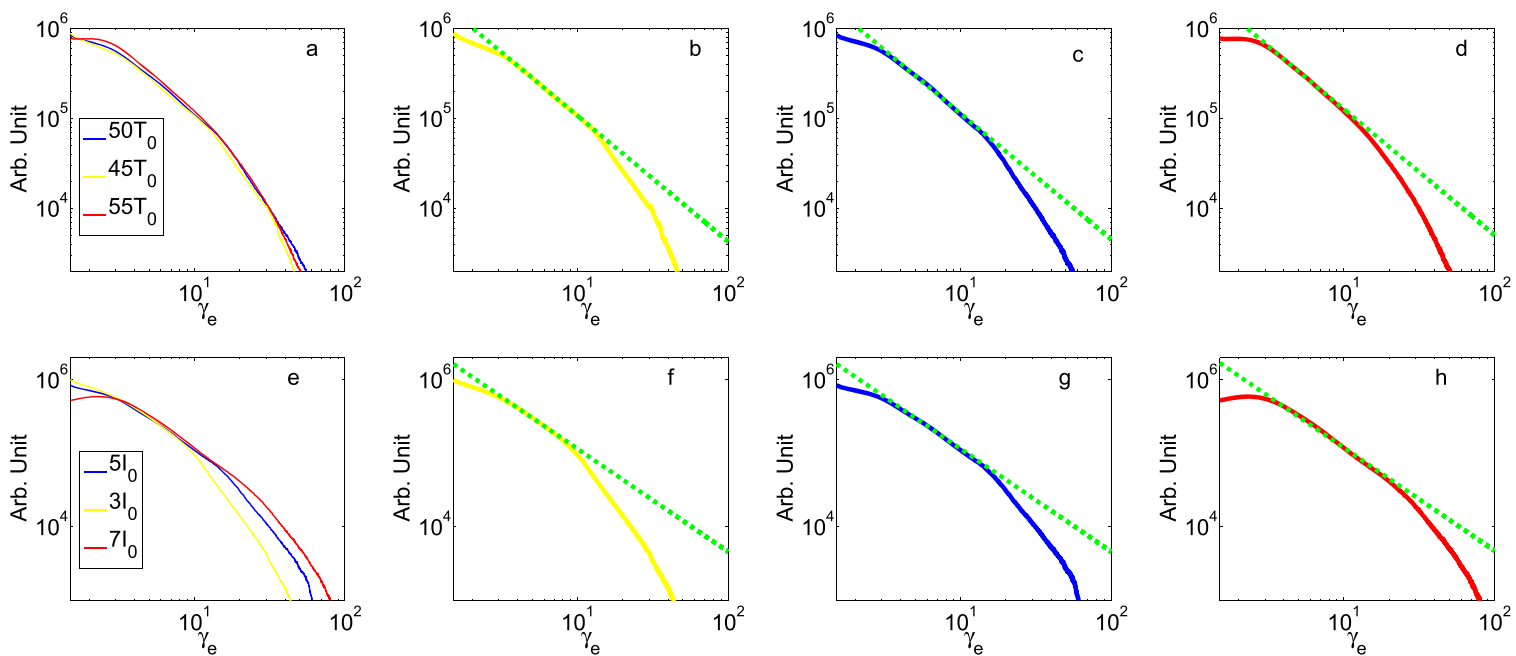

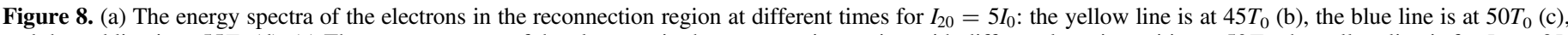

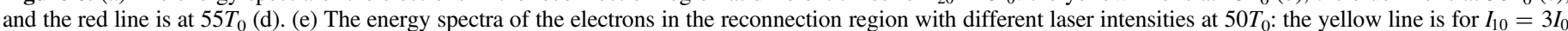

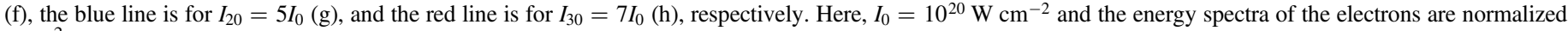
by $\mathrm{m}_{e c}^{2}$.

$\left(p=n k_{B} T_{e}\right)$ to the magnetic pressure $\left(p_{\mathrm{mag}}=B^{2} / 2 \mu \mathrm{m}_{0}\right)$. For $I_{20}=5 \times 10^{20} \mathrm{~W} \mathrm{~cm}^{-2}$, at $t=40 T_{0}$, we calculate the plasma beta $\beta$ in the reconnection cross section in this simulation. We obtain the asymptotic plasma beta is less than unity, with $\beta \sim 0.2-0.3$. Even the maximum beta at the $X$-point is only $\beta \sim 2.5$. According to parameters of plasmas from AGN disks coronae to AGN disks (Frank et al. 2002; Goodman \& Tan 2004; Balbus \& Henri 2008; Goodman \& Uzdensky 2008), the plasma beta is from $1.26 * 10^{-3}$ to 31 . Also in the simulation, the plasma beta is $\sim 0.2-2.5$, in the same range as that in AGN disks and their coronae. Therefore, our simulation could explain certain AGN phenomena. The main difference between the simulation and those in the real astrophysical environment is that the laser driven phenomena have much smaller scales in space and time.

The red, blue, and yellow curves show the reconnection field $E_{z}$ in Figure 7 for laser intensities of $I_{30}=7 \times 10^{20} \mathrm{~W} \mathrm{~cm}^{-2}$, $I_{20}=5 \times 10^{20} \mathrm{~W} \mathrm{~cm}^{-2}$, and $I_{10}=3 \times 10^{20} \mathrm{~W} \mathrm{~cm}^{-2}$, respectively. The maximum of the red line and the blue line is at $t=50 T_{0}$, which corresponds with the pickup ring in Figure $3 \mathrm{C}$ and Figure $3 \mathrm{~B}$. The maximum of the yellow curve is at $t=55 T_{0}$, corresponding to the evolution of the pickup ring in Figure $3 \mathrm{~A}$. Therefore, the reconnection electric field directly affects the evolution of the electron pickup ring.

The energy spectra of the electrons in the reconnection region at a different time for $I_{20}=5 \times 10^{20} \mathrm{~W} \mathrm{~cm}^{-2}$ are shown in Figure 8(a), the yellow line is at $45 T_{0}$ (Figure 8(b)), the blue line is at $50 T_{0}$ (Figure $8(\mathrm{c})$ ) and the red line is at $55 T_{0}$ (Figure $8(d)$ ). The energy spectrum with a power law of $p=1.4$ is presented at different times. At $50 T_{0}$, the range of this energy spectrum is more than at $45 T_{0}$, while it is more than at $55 T_{0}$. This is because, at $50 T_{0}$, the reconnection electric field is highest, and after this time, more energetic electrons are deposited.

Figure 8(e) gives the energy spectra of the electrons in the reconnection region with the different laser intensity at $50 T_{0}$ : the yellow line is for $I_{10}=3 \times 10^{20} \mathrm{~W} \mathrm{~cm}^{-2}$ (Figure 8(f)), the blue line is for $I_{20}=5 \times 10^{20} \mathrm{~W} \mathrm{~cm}^{-2}$ (Figure $8(\mathrm{~g})$ ), and the red line is for $I_{30}=7 \times 10^{20} \mathrm{~W} \mathrm{~cm}^{-2}$ (Figure $8(\mathrm{~h})$ ), respectively. The energy spectrum with a power law of $p=1.4$ is still presented for the different laser intensity, and with the laser intensity increasing, more electrons stay with this energy spectrum. Therefore, the reconnection electric field affects the energy spectra of the electrons. By increasing the reconnection electric field and the laser peak intensity, more electrons are accelerated. The flat energy spectrum with a power law of $p=1.4$ is a characteristic line for MR driven by intense lasers.

In conclusion, the pickup ring of energetic electrons observed in relativistic fast MR driven by ultra-intense femtosecond lasers is investigated by a self-consistent 3D PIC simulation. During MR, two originally axially symmetric O-type configurations of the magnetic flux and plasma current formed by two ultra-intense lasers are separately broken and reconnected. A Sweet-Parker-like Y-type current sheet is created in the merging area. The helical structures of the electron energy density are shown to crash in the MR region and electrons and ions are accelerated by the MR induced parallel electric field. Meanwhile, a "pickup ring" structure in the phase space of $\left(p_{z}, p_{y}\right)$ is formed, indicating a wave particle interaction in the MR process. The energy spectra clearly reveal electron acceleration in the relativistic regime by MR with the energy gain of $\delta \gamma \sim 10-100$. Also, the evolution of pickup ring is affected by different laser peak intensities. The higher the laser peak intensity is, the greater the reconnection electric field will be, and the more visible the electron pickup ring is. The phenomena found in the simulation may be tested experimentally with sub-picosecond sub-petawatt intense laser systems such as LFEX (Shiraga et al. 2011) and OMEGA-EP (Kelly et al. 2006).

This work was supported by Science Challenge Project, No TZ2016005, National Basic Research Program of China (No. 2013CBA01500), the National Natural Science Foundation of China Nos. 10905004, 11220101002, and 11622323, and the Fundamental Research Funds for the Central Universities. Computer runs were performed on the Laohu high performance computer cluster of the National Astronomical Observatories, Chinese Academy of Sciences (NAOC). 


\section{ORCID iDs}

\section{Z. M. Sheng (i) https://orcid.org/0000-0002-8823-9993}

\section{References}

Asano, K., \& Terasawa, T. 2009, ApJ, 705, 1714

Askar'yan, G. A., Bulanov, S. V., Dudnikova, G. I., et al. 1997, PPCF, 39, 137

Balbus, S., \& Henri, P. 2008, ApJ, 674, 408

Biskamp, D. 2000, Magnetic Reconnection in Plasmas (Cambridge: Cambridge Univ. Press)

Brady, P., Ditmire, T., Horton, W., et al. 2009, PhPl, 16, 043112

Chen, M., Sheng., Z., Zheng, J., et al. 2008, Chin. J. Comput. Phys., 25, 45

Dong, Q., Wang, S., Lu, Q., et al. 2012, PhRvL, 108, 215001

Drake, J., Shay, M., Thongthai, W., et al. 2005, PhRvL, 94, 095001

Drake, J., Swisdak, M., Che, H., et al. 2006, Natur, 443, 553

Frank, J., King, A., \& Raine, D. 2002, Accretion Power in Astrophysics (Cambridge: Cambridge Univ. Press)

Fu, X., Lu, Q., \& Wang, S. 2006, PhPl, 13, 012309

Fuchs, J., Malka, G., Adam, J. C., et al. 1998, PhRvL, 80, 1658

Gerbig, D., \& Schlickeiser, R. 2007, ApJ, 664, 750

Ghisellini, G., Celotti, A., \& Lazzati, D. 2000, MNRAS, 313, L1

Goodman, J., \& Tan, J. 2004, ApJ, 608, 108

Goodman, J., \& Uzdensky, D. 2008, ApJ, 688, 555

Homan, D., Kovalev, Y., Lister, M., et al. 2006, ApJL, 642, L115

Kelly, J., Waxer, L., Bagnoud, V., et al. 2006, J. Phys. IV France, 133, 75

Le, A., Egedal, J., Ohia, O., et al. 2013, PhRvL, 110, 135004

Li, C., SŽguin, F., Frenje, J., et al. 2007, PhRvL, 99, 055001

Lin, R., Krucker, S., Hurford, G., et al. 2003, ApJL, 595, L69
Liu, B., Wang, H., Liu, J., et al. 2013, PhRvL, 110, 045002

Lyubarsky, Y., \& Kirk, J. 2001, ApJ, 547, 437

Matteo, T. 1998, MNRAS, 299, L15

McKinney, J., \& Uzdensky, D. 2012, MNRAS, 419, 573

Nakamura, T., \& Mima, K. 2008, PhRvL, 100, 205006

Nakar, E. 2007, PhR, 442, 166

Nilson, P., Willingale, L., Kaluza, M., et al. 2006, PhRvL, 97, 255001

Parker, E. 1957, GeoRL, 62, 509

Ping, Y., Zhong, J., Wang, X., et al. 2014, PhRvE, 89, 031101

Pritchett, P., \& Coroniti, F. 2004, JGR, 109, A01220

Pukhov, A., \& Meyer-ter-Vehn, J. 1996, PhRvL, 76, 3975

Qiao, B., Zhu, S., Zheng, C., et al. 2005, PhPl, 12, 053104

Schlickeiser, R., \& Dermer, C. D. 2000, A\&A, 360, 789

Schlickeiser, R., Pohl, M., \& Vainio, R. 2003, ApJ, 596, 840

Schlickeiser, R., Vainio, R., Böttcher, M., et al. 2002, A\&A, 393, 69

Sheng, Z., \& Meyer-ter-Vehn, J. 1996, PhRvE, 54, 1833

Shiraga, H., Fujioka, S., Nakai, M., et al. 2011, PPCF, 53, 124029

Sweet, P. A. 1958, in IAU Symp. 6, Electromagnetic Phenomena in Cosmical Physics, ed. B. Lehnert (Cambridge: Cambridge Univ. Press), 123

Tanimoto, M., Kato, S., Miura, E., et al. 2003, PhRvE, 68, 026401

Taylor, J. 1986, RvMP, 58, 741

Wagner, U., Tatarakis, M., Gopal, A., et al. 2004, PhRvE, 70, 026401

Wan, W., Lapenta, G., \& Delzanno, G. 2008, PhPl, 15, 032903

Waxman, E. 2006, PPCF, 48, B137

Wu, C., \& Davidson, R. 1972, JGR, 77, 5399

Yamada, M., Kulsrud, R., \& Ji, H. 2010, RvMP, 82, 603

Yan, X., Lin, C., Sheng, Z., et al. 2008, PhRvL, 100, 135003

Zenitani, S., \& Hoshino, M. 2001, ApJL, 562, L63

Zhang, B., \& Mészáros, P. 2004, IJMPA, 19, 2385

Zhong, J., Li, Y., Wang, X., et al. 2010, NatPh, 6, 984 